\title{
Translational advancements in transdermal and mucosal delivery
}

\author{
Emmanuel A. Ho ${ }^{1,2} \cdot$ Ryan F. Donnelly ${ }^{3}$
}

Accepted: 1 February 2022 / Published online: 8 February 2022

c) Controlled Release Society 2022

Parenteral route (also referred to as injection route) of drug administration has many advantages including rapid systemic effects and the ability to deliver drugs locally to a given region. Unfortunately, the major challenge with parenteral drug administration is low patient acceptance which stems from the fear and pain of needles. As a result, alternative routes of drug administration are desired by most patients. In this special issue, we have highlighted some of the major advancements in the fields of transdermal and mucosal drug delivery and its rapidly growing potential in improving global health.

Being the largest and most accessible organ, the skin is an attractive target for drug delivery. Years of research in the area of skin permeation culminated with the regulatory approval of the first transdermal patch for the delivery of the antiemetic drug scopolamine in 1979 [1]. Since then, the delivery of a wide range of therapeutics across the skin has progressed considerably with the development of various innovative drug delivery strategies. However, the efficient barrier function of the stratum corneum presents a considerable challenge to the delivery of therapeutics into and across the skin. As a result, numerous strategies have been applied to enhancing transdermal and intradermal drug delivery. Considering the progress made in terms of research into novel enhancement methodologies, it could be hypothesized that we are indeed only at the beginning of fully exploiting the commercial potential of the transdermal drug delivery market. To ensure continued success using the transdermal

Emmanuel A. Ho

Emmanuel.ho@uwaterloo.ca

Ryan F. Donnelly

R.Donnelly@qub.ac.uk

1 Faculty of Science, School of Pharmacy, University of Waterloo, 10A Victoria St. S., Room 4002, Kitchener, ON N2G 1C5, Canada

2 Waterloo Institute for Nanotechnology, Waterloo, Canada

3 School of Pharmacy, Queen's University Belfast, 97 Lisburn Road, Belfast BT9 7BL, UK route for drug delivery, the only solution appears to be the exploitation of active technologies to overcome the limiting factor of the skin barrier. There is substantial evidence of enhanced skin permeability using these techniques for a wealth of drug compounds and the task is now to translate this into successful marketed products. There are lessons to be learnt from the few approvals granted by the United States Food and Drug Administration (FDA) over the last number of years and the eventual fate of these products, alongside those product ideas which didn't progress as far as market, whether that be for financial reasons or concerns over their safety and/or technical complexity. This special issue considers a range of novel technologies with translational potential including contributions from Xiao Yu Wu's group (University of Toronto), Chin-Ping Kung (University College London) and collaborators, Davide Brambilla (Université de Montreal) and collaborators, M. Begona DelgadoCharro (University of Bath) and team, Raghu Raj Singh Thakur (Queen's University Belfast) and collaborators, and Suohui Zhang (Chinese Academy of Sciences) and team.

The mucosal membrane is a dynamic and complex layer that lines a variety of cavities such as the GI tract, oronasal cavity, pulmonary tract, and the female reproductive tract. The mucosal membrane is involved in the absorption and secretion of a wide range of molecules and is an important target for vaccine development and the treatment of diseases such as cancer, sexually transmitted infections, and pulmonary disorders. Despite advancements made in the field of mucosal drug delivery, there are still challenges and barriers to developing effective mucosal therapeutics for both local and systemic applications. In this special issue, contributions from world experts discuss the challenges/barriers encountered along with novel strategies to overcome these obstacles to develop a successful therapeutic. This includes articles submitted by Maria José Alonso's group (Universidade de Santiago de Compostela), Olivia Merkel (LudwigMaximilians-Universitat Munchen) and collaborators, Laura Ensign (Johns Hopkins University) and team, and Geoffrey Mortuaire (Université de Lille) and collaborators. 
Transdermal and mucosal drug delivery are unique and very exciting fields. There is so much that we still need to learn in terms of understanding how these barriers function and how we can optimally design strategies to improve delivery of therapeutics across these barriers. We hope that this special issue will appeal to those that are new to transdermal and mucosal drug delivery while continuing to excite those that are already pushing the boundaries of the field to the next level.

\section{Reference}

1. Pastore MN, Kalia YN, Horstmann M, Roberts MS. Transdermal patches: History, development and pharmacology. Br J Pharmacol. 2015;172:2179-209.

Publisher's Note Springer Nature remains neutral with regard to jurisdictional claims in published maps and institutional affiliations. 